\title{
PREVENÇÃO CONTRA DANOS PROVOCADOS POR SURTO DE TENSÃO DEVIDO ÀS DESCARGAS ATMOSFÉRICAS ATRAVÉS DE SISTEMA ELETRÔNICO MICROCONTROLADO
}

\section{LIGHTNING PROTECTION EMBEDDED SYSTEM FOR ELECTRONIC EQUIPMENTS}

\section{Anderson Soares André}

Universidade do Sul de Santa Catarina (UNISUL), e-mail: anderson.andré@unisul.br

\author{
João Luiz Alkaim
}

Universidade do Sul de Santa Catarina (UNISUL), e-mail: joao.alkaim@unisul.br

\section{Julibio David Ardigo}

Universidade do Sul de Santa Catarina (UNISUL), e-mail: julibio.ardigo@unisul.br

\section{Sérgio Luciano Avila}

Universidade do Sul de Santa Catarina (UNISUL), e-mail: sergio.avila@unisul.br

\section{Bruno Filippe Espindola}

Universidade do Sul de Santa Catarina (UNISUL), e-mail: bruno_fespindola@hotmail.com

Resumo: Este artigo apresenta um sistema microcontrolado, com interface computacional, que permite fazer a aquisição de dados provenientes do Sistema de Informações Integradas Baseado no Sistema de Detecção de Descargas Atmosféricas (SIDDEM), de forma a obter informações das descargas atmosféricas ocorridas na região da Subestação localizada em Palhoça/SC, de propriedade da Eletrosul Centrais Elétricas S.A. Tais dados subsidiam as tomadas de decisão do sistema desenvolvido a fim de proteger equipamentos eletrônicos de danos que possam ser causados por surto de tensão devido a estas descargas, desconectando-os da rede elétrica e mantendo-os em funcionamento através de um no-break. O local foi escolhido em função de sua facilidade de acesso, mas o sistema desenvolvido pode ser facilmente utilizado em outros pontos de interesse da Eletrosul Centrais Elétricas S.A.

Palavras-chave: Descarga Atmosférica. Proteção. Microcontrolador.

Abstract: This paper presents an embedded system, with computational interface, which allows the acquisition of data coming from the Integrated Information System Based on the System of Lightning Detection (SIDDEM - Portuguese acronym), in order 
to obtain information of lightning occurred in the area of substation located in Palhoça, which belongs to Eletrosul Centrais Elétricas S.A. Such data support the decision-making system developed to protect electronic equipment from damage that may be caused by power surge due to these discharges, disconnecting electrical network and keeping them running through an UPS. The site was chosen due to its ease of access, but the system can be easily used in other points of interest of Eletrosul Centrais Elétricas S.A.

Key-words: Lightning. Protection. Electronic. Microcontroler. 


\section{INTRODUÇÃO}

A evolução registrada nos equipamentos de proteção contra descargas atmosféricas e sua disseminação têm proporcionado substancial redução nos eventos que se traduzem em danos aos equipamentos eletroeletrônicos conectados às redes elétricas durante as tempestades. Entretanto, é sabido que toda esta tecnologia não é capaz de eliminar $100 \%$ dos riscos da ocorrência de faltas no sistema elétrico e outras áreas, o que incrementa o interesse sobre tal fenômeno devido aos prejuízos causados às concessionárias de energia elétrica, agricultura e diversos outros órgãos prestadores de serviços à sociedade. Este interesse, por sua vez, despertou a necessidade para o início dos estudos climatológicos sobre descargas atmosféricas no Brasil, cujos trabalhos iniciais datam da década de 90 , conforme Naccarato (2006).

Com a finalidade de atender a uma série de necessidades ligadas à esta área do conhecimento, no ano de 2006 foi implantado o projeto SIDDEM no cone sul brasileiro, envolvendo os estados do Rio Grande do Sul, Santa Catarina e Mato Grosso do Sul, e patrocinado por um conjunto de empresas, tais como: Eletrosul Centrais Elétricas S.A, Tractebel Energia, Companhia Estadual de Energia Elétrica - Rio Grande do Sul (CEEE), Centrais Elétricas de Santa Catarina (CELESC), Empresa de Pesquisa Agropecuária e Extensão Rural de Santa Catarina (EPAGRI) entre outras.

O Sistema de Informações Integradas Baseado no Sistema de Detecção de Descargas Atmosféricas (SIDDEM) é composto de dois sistemas de deteç̧ões de descargas atmosféricas e de um sistema de informações via web, queenvia dadosàs unidades usuárias para auxílio no monitoramento e previsão do tempo, diminuindo substancialmente os prejuízos provocados por fenômenos climáticos, devido ao fornecimento antecipado de informações.

Com base nas informações recebidas do sistema supracitado, o equipamento desenvolvido é capaz de verificar a existência de riscos à instalação protegida através de cálculos baseados no modelo eletrogeométrico, desconectando-os da rede elétrica enquanto as descargas estiverem dentro da área conhecida como raio de atração. Uma vez que cessem as descargas dentro desta área, os equipamentos protegidos voltam a ser conectados à rede elétrica.

\section{DESCARGAS ATMOSFÉRICAS}

Sempre que uma nuvem passa através de outra, devido ao atrito registrado, ocorre troca de cargas elétricas entre as mesmas fazendo com que estas fiquem "carregadas". Assim, sempre que duas ou mais nuvens carregadas estão próximas, uma diferença de potencial elétrico é gerada entre as mesmas. Além da diferença de potencial existente entre as nuvens, existe também uma tensão entre a nuvem carregada e o solo. Como conseqüência deste fenômeno, existe um campo elétrico que permeia todo o espaço envolvido. Quando este campo elétrico assume um valor maior que a rigidez dielétrica 
do ar, uma corrente elétrica flui de uma nuvem para outra, ou de uma nuvem para o ar, se dissipando, ou ainda, da nuvem para o solo, gerando uma descarga atmosférica, popularmente conhecida como "raio".

De acordo com Visacro Filho (2005) existem basicamente dois tipos de descargas atmosféricas, que são classificadas de acordo com o trajeto percorrido pela corrente elétrica. As descargas em nuvem são aquelas que se iniciam dentro da nuvem. $O$ destino final de tal descarga pode estar dentro da própria nuvem, sendo chamada neste caso de descarga intra-nuvem; pode estar em outra(s) nuvem(ns) (descarga nuvem-nuvem) ou o destino final pode estar fora da nuvem, mas sem atingir o solo e sem atingir outra nuvem, ou seja, são descargas para o ar.

A segunda classificação possível faz referência às descarga entre nuvem e solo, que pode ser do tipo nuvem-solo ou de solo-nuvem, conforme o sentido do movimento da carga que a origina. Então o raio pode ter início na superfície da nuvem ou no chão.

\subsection{CONCEITOS RELEVANTES PARA O PROJETO}

A fim de tornar mais claros alguns conceitos aplicados ao projeto recorreu-se, mais uma vez, às informações constantes em Visacro Filho (2005), como segue:

a) raio ou descarga atmosférica: conceito amplo correspondente ao fenômeno integral envolvido na evolução e fechamento do canal de descarga, incluindo o fluxo da(s) corrente(s) de retorno e as demais manifestações elétricas, visuais e sonoras;

b) relâmpago: efeito luminoso perceptível visualmente, decorrente do aquecimento do canal de descarga devido ao fluxo da corrente de retorno pelo canal;

c) trovão: efeito sonoro relacionado à descarga atmosférica. É causado pelo brusco deslocamento de ar circunvizinho ao canal de descarga, que se expande em decorrência do aquecimento gerado pelo fluxo da corrente no canal;

d) descarga elétrica: fluxo de cargas a partir de um objeto carregado eletricamente. Pode corresponder a processo disruptivo em meio isolante (tipo faísca) ou ao simples descarregamento através do meio condutor. $O$ termo é algumas vezes empregado para designar o fenômeno descarga atmosférica. Tal emprego não é considerado adequado, pois não confere caráter de especificidade ao significado de descarga elétrica;

e) canal precursor de descarga: corresponde ao canal ionizado que evolui por passos, da ordem de $50 \mathrm{~m}$, correspondentes a descargas elétricas (rupturas) sucessivas no ar, que ocorrem a cada intervalo da ordem de $50 \mu$ s. A evolução do canal pode fechar um percurso ionizado entre nuvem e solo (no caso de 
descargas nuvem-solo), que é seguido pelo fluxo da corrente de retorno pelo canal formado. Pode ter seu percurso predominantemente ascendente, recebendo nesse caso respectivamente a designação de canal ascendente ou descendente;

f) canal descendente: refere-se a um canal ionizado configurado a partir da disrupção do ar e que se propaga descendentemente a partir da nuvem de tempestade. O canal se constitui através de descargas elétricas sucessivas, que ocorrem por passos a partir da nuvem, e evolui em direção ao solo. Seu percurso preferencial tem a direção vertical, embora apresente tortuosidades, e pode incluir ramificações;

g) canal ascendente: refere-se a um canal ionizado, configurado a partir da disrupção do ar, que se propaga ascendentemente a partir do solo. O canal se constitui através de descargas elétricas sucessivas, que ocorrem por passos, e evolui ascendendo numa direção predominantemente vertical;

h) canal de descarga: canal ionizado correspondente ao percurso integral que conecta os pontos entre os quais ocorre a descarga. Possui um núcleo ionizado, de boa condutividade, cujo raio é da ordem de alguns centímetros. O núcleo é envolto por um envelope de Corona de vários metros, onde ficam acumuladas as cargas elétricas. O canal é constituído quando, eventualmente, o canal precursor descendente encontra um canal precursor ascendente, ao fechar o percurso de descarga através de um salto final. A conexão desses canais resulta no fluxo da corrente de retorno pelo canal de descarga constituído. No caso da descarga nuvem-solo, o percurso tem a ordem de vários quilômetros entre a nuvem e o solo;

i) corrente de retorno: corrente que flui pelo percurso ionizado correspondente ao canal de descarga após o seu fechamento e que corresponde ao descarregamento deste. Tal corrente é constituída basicamente pela neutralização das cargas armazenadas no envelope de Corona que envolve o núcleo do canal de descarga;

j) corrente de recarregamento do canal de descarga: corrente de valor reduzido (usualmente de algumas centenas de ampères) correspondente ao deslocamento de cargas negativas da nuvem para o canal remanescente de uma descarga de retorno negativa anterior. Logo após o fluxo de uma corrente de retorno, o canal tende a se dissipar. Em alguns casos (de 70\% a $80 \%$ ), ocorrem processos disruptivos na parcela superior do canal que o conectam a outro centro de cargas negativas na nuvem. Neste caso, as cargas desse centro se deslocam para o canal na forma de uma corrente de baixa intensidade. Essa corrente é responsável pelo recarregamento de uma extensão do canal de descarga antes que o mesmo se dissipe e, eventualmente, pode ser capaz de reacendê-lo e habilitá-lo a uma descarga de retorno posterior. Esta pode fechar 
no mesmo tempo ponto de incidência (caso de uso integral do canal anterior) ou num ponto do solo próximo ao da descarga anterior, a distâncias máximas de alguns quilômetros (mais usualmente limitadas a $2 \mathrm{~km}$ ), no caso de uso de apenas parte do canal anterior;

k) processo de recarregamento do canal de descarga: corresponde ao evento associado ao fluxo da corrente de recarregamento do canal de uma descarga anterior, em dissipação após o fluxo de uma corrente de retorno. A corrente de recarregamento promove um efeito visual similar a um dardo se deslocando ao longo do canal, a partir da nuvem. Daí derivou a designação em língua inglesa para o evento. $O$ recarregamento pode ocorrer no percurso integral do canal de descarga anterior, sendo seguido do fluxo de uma corrente de retorno subseqüente. Nesse caso, a descarga subseqüente se completa no mesmo ponto de incidência da descarga anterior. Nos casos em que a parte inferior do canal de descarga anterior já havia se dissipado, ocorre o carregamento de apenas uma parcela do canal. Pode haver a evolução desse canal, por passos, até o seu fechamento próximo ao solo, que é seguido pelo fluxo da corrente de retorno. Nessa última situação, o ponto de incidência da descarga subseqüente pode se localizar a distâncias significativas daquele da primeira descarga. Tal distância pode ter a ordem de alguns quilômetros (usualmente até $2 \mathrm{~km}$ );

I) processo de conexão: estágio final do processo de evolução dos canais ascendente e descendente, que corresponde a conexão dos mesmos. Desta conexão fica constituído o canal de descarga, no qual haverá o fluxo da corrente de retorno;

m) distância de salto final: distância crítica entre os canais descendente e ascendente, a partir da qual ocorre o fechamento do percurso entre os mesmos, através de uma descarga elétrica disruptiva final. Aparentemente, a descarga final se origina no canal ascendente. Tal distância depende, sobretudo, da carga acumulada no canal precursor da descarga;

n) canal piloto de descarga: canal estabelecido junto a um eletrodo ou na extremidade de um canal ionizado (ascendente e descendente), o qual precede a formação do salto da descarga elétrica plena;

o) raio de atração: distância crítica entre o canal descendente e a estrutura terrestre a partir da qual tem-se a expectativa do fechamento do percurso entre o canal descendente e o canal ascendente, que se originou nessa estrutura. Termo muito utilizado na literatura de proteção contra descargas para designar a distância máxima entre a extremidade de um eventual canal descendente e a estrutura terrestre que asseguraria o processo de conexão, para cada valor de carga armazenada no canal (ou correspondente valor de pico da corrente de retorno associada). Tal distância é usualmente inferior a distância de indução do canal ascendente; 
p) distância de indução do canal ascendente: distância crítica entre o canal descendente e a estrutura terrestre a partir da qual se dá o surgimento de canal(is) ascendente(s);

q) descarga atmosférica plena: referência à descarga atmosférica no sentido mais restrito a fase de fluxo das correntes de retorno. Corresponde ao conjunto de descargas de retorno envolvidas após o fechamento do canal. Assim, a descarga plena pode ser constituída de uma ou mais descargas pelo canal, dependendo da existência de descargas subseqüentes;

r) descarga de retorno: evento associado ao fluxo da corrente de retorno correspondente a uma descarga elétrica pelo canal de descarga. Uma descarga atmosférica plena pode incluir várias descargas de retorno ou uma única descarga de retorno;

s) primeira descarga de retorno: primeira descarga de retorno de uma descarga atmosférica plena;

t) descarga(s) de retorno subseqüente(s): conjunto de descargas de retorno subseqüentes à primeira descarga de retorno de um flash, ou uma destas descargas de retorno;

u) descarga direta: refere-se ao evento associado à incidência de uma descarga diretamente sobre uma vítima (ser, estrutura, objeto, etc.);

v) descarga indireta, lateral ou próxima: refere-se ao evento associado a uma descarga que incide próxima à vítima (ser, estrutura ou sistema afetado), que pode ser capaz de gerar efeitos na mesma, em decorrência dos campos eletromagnéticos gerados.

\subsection{MODELO ELETROGEOMÉTRICO DE INCIDÊNCIA}

O modelo eletrogeométrico utilizado no sistema desenvolvido foi fundamentado no raio de atração. Para a subestação de Palhoça podem ser desenvolvidas duas metodologias: a primeira totalmente centrada no raio de atração (RA) e a segunda no método das esferas rolantes, que é uma situação particular do modelo de incidência, como apresentadas por Visacro Filho (2005) Tais metodologias são brevemente discutidas a seguir.

\subsubsection{Primeira metodologia}

A primeira metodologia fundamenta-se no conceito do raio de atração, que se constitui na distância estimada entre um canal descendente e a estrutura terrestre, a partir da qual ocorrerá eventual fechamento do percurso pela conexão entre o canal descendente e o canal ascendente que se origina na estrutura terrestre. Admite-se que, caso tal distância seja alcançada, haja grande probabilidade de incidência na estrutura. 
Através de experimentos laboratoriais com modelos em escala reduzida, de registros fotográficos e de filmagens da incidência de descargas reais em torres instrumentadas, foram desenvolvidas relações empíricas que relacionam o raio de atração com o valor de pico da corrente de descarga (Ip). A maior parte destas expressões tem a forma apresentada na equação (1), sendo A e B constantes empíricas, e fornecem resultados muito similares.

$$
R a=A \cdot I p^{B}
$$

De acordo com Brown e Whitehead (1969) o raio de atração pode ser calculado por:

$$
R a=7,1 . I p^{0,75}
$$

Já Love (1973) e Anderson (1982) propõem a expressão:

$$
R a=10 . I p^{0,66}
$$

De acordo com Armstrong e Whitehead (1968), este valor pode ser obtido por:

$$
R a=6,7 \cdot I p^{0,8}
$$

Finalmente, de acordo com a norma NBR5419 da Associação Brasileira de Normas Técnicas (2001), o raio de atração vem de:

$$
R a=2,0 . I p+30 .\left(1-e^{-I p}\right)
$$

Em todas as expressões propostas, o raio de atração é definido em metros $(m)$ e a corrente em quilo-Amperes (kA). Através de todas as equações apresentadas acima, fica claro que o raio de atração depende da intensidade da corrente da descarga.

O quadro 1 apresenta os valores de raio de atração obtidos por simulação para as metodologias propostas por Anderson (1982) e pela Associação Brasileira de Normas Técnicas (2001) conforme a NBR5419. 


\begin{tabular}{|c|c|c|}
\hline Corrente de Pico (kA) & $\begin{array}{c}\text { Raio de Atração de Acordo } \\
\text { com a NBR5419 }\end{array}$ & $\begin{array}{c}\text { Raio de Atração de Acordo } \\
\text { com Anderson (1982) }\end{array}$ \\
\hline 05 & 40 & 29 \\
\hline 10 & 50 & 46 \\
\hline 15 & 60 & 60 \\
\hline 20 & 70 & 72 \\
\hline 30 & 90 & 94 \\
\hline 50 & 130 & 132 \\
\hline 100 & 230 & 209 \\
\hline
\end{tabular}

Quadro 1: Comparação Entre Diferentes Abordagens Para o Raio de Atração.

Em função da natureza empírica das constantes utilizadas, percebe-se diferenças apreciáveis nos valores obtidos para pequenas correntes, entretanto, para correntes acima de $15 \mathrm{kA}$, que correspondem à valores de maior interesse para a proteção de equipamentos, os raios de atração obtidos assemelham-se bastante.

Segundo Visacro Filho (2005), no aprimoramento contínuo da metodologia do raio de atração, foram desenvolvidas expressões que tentam computar outros efeitos que exercem influência na sua definição, incluindo características da estrutura a ser atingida, como sua altura $(\mathrm{H})$.

\begin{tabular}{|c|c|c|c|}
\hline $\begin{array}{c}\text { Corrente de } \\
\text { Pico (kA) }\end{array}$ & $\begin{array}{c}\text { Raio de Atração de Acordo } \\
\text { com Anderson (1982) }\end{array}$ & Altura $(\mathrm{m})$ & $\begin{array}{c}\text { Raio de Atração de Acordo } \\
\text { com Eriksson(1987) }\end{array}$ \\
\hline & & 10 & 13 \\
5 & 29 & 40 & 20 \\
& & 80 & 32 \\
& & 10 & 51 \\
\hline 10 & 46 & 20 & 20 \\
& & 40 & 32 \\
\hline
\end{tabular}




\begin{tabular}{|l|c|c|c|}
\hline & & 10 & 31 \\
20 & & 20 & 50 \\
& 72 & 40 & 79 \\
& & 80 & 125 \\
\hline 50 & 132 & 10 & 57 \\
& & 20 & 91 \\
& & 40 & 145 \\
\hline
\end{tabular}

Quadro 2: Efeito da altura da estrutura no valor do raio de atração.

A equação 6, desenvolvida por Eriksson (1987), mostra esta consideração para proteção de linhas de transmissão:

$$
R_{a}=H^{\left(0,6+2 . I_{p} \cdot 0^{-4}\right)} \cdot I_{p}^{(0 . \varangle)}
$$

O quadro 2 permite fazer uma comparação dos valores de raio de atração obtidos utilizando-se a metodologia proposta por Anderson (1982) e por Eriksson (1987), ilustrando o efeito da altura da estrutura.

Em função dos resultados obtidos e para efeitos de simplificação adotou-se neste projeto a metodologia que se encontra na norma NBR-5419 que relaciona o raio de atração com a corrente de pico (Ip), em kA, da descarga atmosférica e é definida pela equação (5) (ASSOCIAÇÃO BRASILEIRA DE NORMAS TÉCNICAS, 2001).

\subsection{CARACTERIZAÇÃO DOS NÍVEIS DE PROTEÇÃO SEGUNDO NBR 5419}

O quadro 3 apresenta nova correlação entre grandezas envolvidas, incluindo a corrente mínima que o Sistema de Proteção contra Descargas Atmosféricas (SPDA) deve blindar segundo os preceitos propostos na norma brasileira.

\begin{tabular}{|c|c|c|c|}
\hline $\begin{array}{c}\text { Nível de } \\
\text { Proteção }\end{array}$ & Tipo de Estrutura & $\begin{array}{c}\text { Corrente que o SPDA } \\
\text { deve Blindar }(k A)\end{array}$ & $\begin{array}{c}\text { Raio de } \\
\text { Atração } \\
(\mathrm{m})\end{array}$ \\
\hline
\end{tabular}




\begin{tabular}{|c|c|c|c|}
\hline I & $\begin{array}{c}\text { Instalações de risco como } \\
\text { refinarias, postos de combustível, } \\
\text { usinas de energia elétrica e } \\
\text { estações de comunicação. }\end{array}$ & 3,7 & 20 \\
\hline II & $\begin{array}{c}\text { Locais públicos como escolas, } \\
\text { igrejas, hospitais, bancos e lojas. }\end{array}$ & 6,1 & 30 \\
\hline III & $\begin{array}{c}\text { Residências, indústrias, } \\
\text { fazendas e estabelecimentos } \\
\text { agropecuários em madeira. }\end{array}$ & 10,6 & 60 \\
\hline IV & $\begin{array}{c}\text { Fazendas e estabelecimentos } \\
\text { agropecuários com menor } \\
\text { condição de risco. }\end{array}$ & 16,5 & 45 \\
\hline
\end{tabular}

Quadro 3: Caracterização dos Níveis de Proteção de Segundo a NBR 5419 Fonte: Associação Brasileira de Normas Técnicas (2001).

Os diferentes níveis de proteção apresentados no quadro 3 estão ligados aos tipos de instalações que se deseja proteger.

\subsubsection{Segunda metodologia}

Segundo Visacro (2005) outra metodologia, um pouco mais elaborada, é desenvolvida na perspectiva das possíveis posições alcançadas pela extremidade do canal descendente em sua aproximação da estrutura, sendo designada: Método das Esferas Rolantes.

A cada amplitude de corrente está associado um raio de atração específico que define uma esfera centrada em cada posição admitida para a extremidade do canal descendente. Tal esfera é deslocada (rolada) sobre a superfície da estrutura cuja proteção se projeta. Assim, o centro da esfera cobre todas as possíveis posições que a extremidade de um eventual canal descendente poderia ocupar ao se aproximar da estrutura.

O Método das Esferas Rolantes constitui-se numa aplicação particular do Modelo Eletrogeométrico de Incidência.

\section{PROTÓTIPO}

Baseado no que foi exposto anteriormente, um protótipo do sistema de proteção foi desenvolvido em laboratório e testado na Subestação de Palhoça. Para tanto, utilizou-se a abordagem de determinação do raio de atração baseado nos pressupostos apresentados na NBR 5419. Tal valor permite verificar se uma dada descarga atmosférica pode ou não causar danos aos equipamentos eletrônicos instalados no site protegido. No teste piloto foram protegidos somente os equipamentos de informática de uma das salas da subestação. 
A janela de monitoramento de descargas atmosféricas utilizadas no teste piloto compreende uma área com raio de $10 \mathrm{~km}$ a partir da subestação cujas coordenadas geodésicas são: $27^{\circ} 38^{\prime} 33,00635^{\prime \prime}$ de latitude sul e $48^{\circ} 41^{\prime} 28,57578^{\prime \prime}$ de longitude oeste. Desta forma, os limites da área de proteção ficam definidos como sendo:

\begin{tabular}{|l|c|}
\hline \multicolumn{1}{|c|}{ Limite } & Coordenada \\
\hline Superior & $27^{\circ} 33^{\prime} 03,15^{\prime \prime} \mathrm{S}$ \\
Inferior & $27^{\circ} 43^{\prime} 53,96^{\prime \prime} \mathrm{S}$ \\
Lateral Esquerdo & $48^{\circ} 47^{\prime} 33,41^{\prime \prime} \mathrm{O}$ \\
Lateral Direito & $48^{\circ} 35^{\prime} 21,74^{\prime \prime} \mathrm{O}$ \\
\hline
\end{tabular}

Quadro 4: Limites da Área de Proteção do Site em Estudo.

Uma vez definidos tais limites, foi possível desenvolver o software e os dispositivos eletrônicos do protótipo, conforme detalhado nos itens que se seguem.

\subsection{SOFTWARE DE CONTROLE}

O software de controle deve atender diversas especificações ligadas às limitações impostas pelos sistemas com os quais o protótipo deve trocar informações. As principais são:

ser capaz de fazer a aquisição dos dados da EPAGRI, provenientes da rede Sistema de Informações Integradas Baseado no SIDDEM, através dos protocolos TCP/IP;

realizar os cálculos necessários para a determinação do raio de atração, a distância entre cada descarga atmosférica e a Subestação da Eletrosul (ponto piloto) e, com base nos cálculos anteriores, definir se existe perigo de danos aos equipamentos protegidos;

dispor de porta de comunicação tipo serial RS-232 para comunicação entre o software residente no computador de controle e o hardware do protótipo.

O fluxograma simplificado de funcionamento do software pode ser visto na Figura 1, tendo sido desenvolvimento na interface gráfica IDE da Borland Delphi 7. 


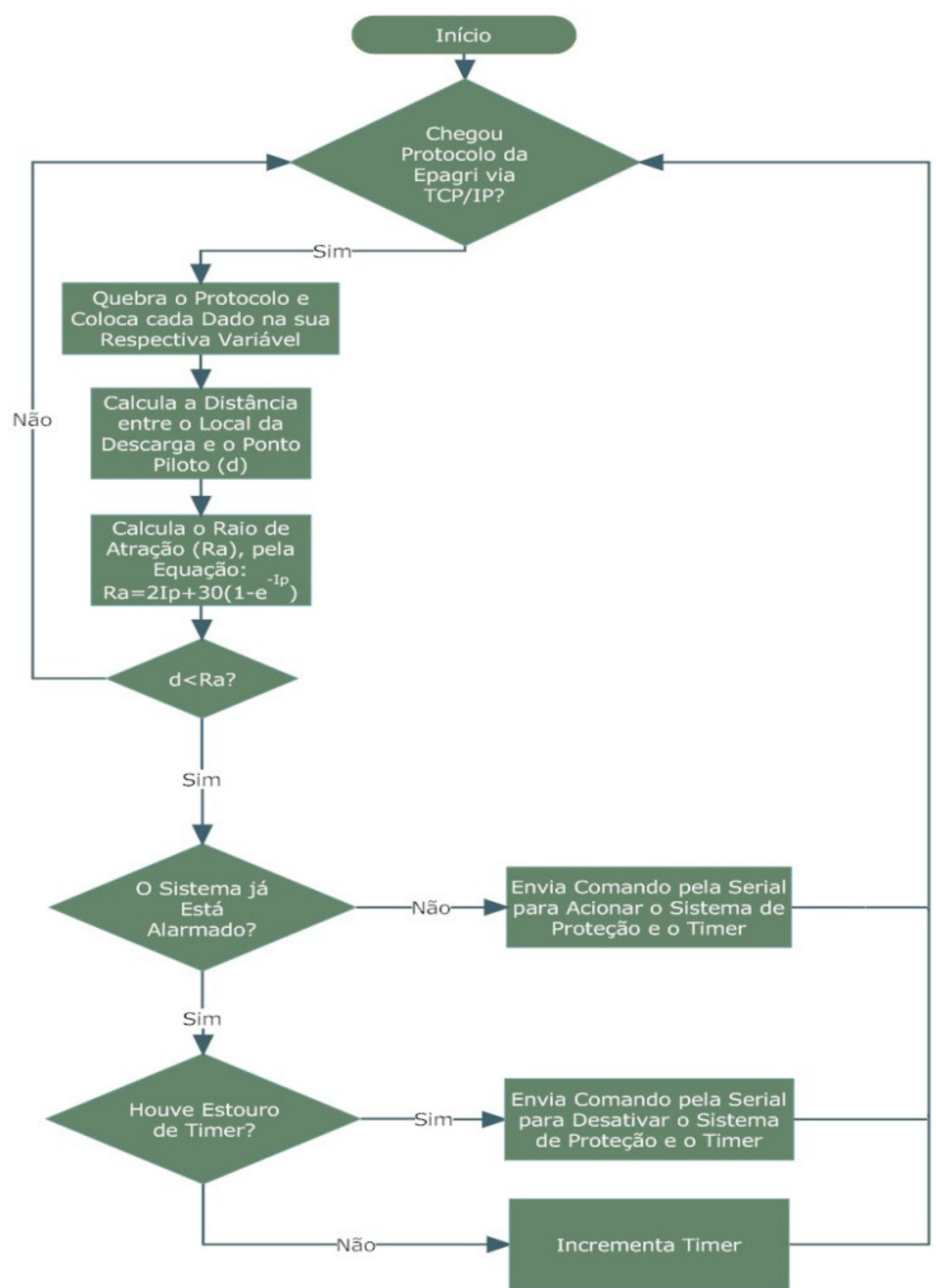

Figura 1: Fluxograma Simplificado de Funcionamento do Software. 
No primeiro passo, o software recebe o protocolo de dados da EPAGRI no formato UALF 0 (Universal Ascii Lightning Format), fornecido pela empresa Vaisala, fabricante dos sensores de descarga atmosféricas do projeto SIDDEM.

Trata-se de um protocolo que inclui todos os parâmetros de uma descarga atmosférica, separados por $<$ tab $>$, ou seja, (0x09), conforme mostra a quadro abaixo.

\begin{tabular}{|lrllllllllllllll|}
\hline 0 & 2007 & 3 & 28 & 21 & 39 & 1 & 509465750 & 0.0000 & 0.0000 & 0 & 0 & 0 & 0 \\
0.00 & 0.00 & 0.00 & 0.00 & 0.0 & 0.0 & 0.0 & 0 & 0 & 0 & 0 & & \\
\multicolumn{1}{c|}{ Quadro 5: Formato de Dados das Descargas. }
\end{tabular}

O protocolo UALF 0 define rigidamente o significado de cada um dos parâmetros apresentados, como se observa no quadro 6.

\begin{tabular}{|c|c|}
\hline Campo & Definição \\
\hline 1 & Versão do Protocolo UALF, de 0 a 1 \\
\hline 2 & Ano, de 1970 a 2032 \\
\hline 3 & Mês, com Janeiro como sendo 1 e Dezembro como sendo 12 \\
\hline 4 & Dia do mês, de 1 a 31 \\
\hline 5 & Hora, de 0 a 23 \\
\hline 6 & Minuto, de 0 a 59 \\
\hline 7 & Segundo, de 0 a 59 \\
\hline 8 & Nanossegundo, de 0 a 999999999 \\
\hline 9 & $\begin{array}{l}\text { Latitude do local calculado em graus decimais, com } 4 \text { casas decimais, } \\
\text {-90.0 a } 90.0\end{array}$ \\
\hline 10 & $\begin{array}{l}\text { Longitude do local calculado em graus decimais, com } 4 \text { casas } \\
\text { decimais, }-180.0 \text { a } 180.0\end{array}$ \\
\hline 11 & Corrente de pico estimada em kA, de -9999 a 9999 \\
\hline 12 & Multiplicidade para dados de flash (1 a 99) ou 0 para strokes \\
\hline 13 & Números de sensores que participaram da solução, de 2 a 99 \\
\hline 14 & Grau de liberdade para aperfeiçoar a localização, de 0 a 99 \\
\hline 15 & Ângulo da Elipse com orientação horária, de 0 a 180.0 graus \\
\hline 16 & Semi-eixo maior da Elipse, em quilômetros, de 0 a 50.0Km \\
\hline
\end{tabular}




\begin{tabular}{|c|c|}
\hline 17 & Semi-eixo menor da Elipse, em quilômetros, de 0 a 50.0Km \\
\hline 18 & Valor qui-quadrado para otimização da localização, de 0 a 999.99 \\
\hline 19 & $\begin{array}{l}\text { Tempo de subida da forma de onda, em microssegundos, de } 0 \text { a } \\
99.9\end{array}$ \\
\hline 20 & $\begin{array}{l}\text { Tempo entre o pico da forma de onda e o zero, em microssegundos, } \\
\text { de } 0 \text { a } 999.99\end{array}$ \\
\hline 21 & Taxa máxima de subida da forma de onda, em kA/ $\mu$ seg, de 0 a999.9 \\
\hline 22 & $\begin{array}{l}\text { Indicador do tipo de descarga. } 1 \text { para descargas nuvem-nuvem e } 0 \\
\text { para descargas nuvem-solo }\end{array}$ \\
\hline 23 & $\begin{array}{l}\text { Indicador de ângulo. } 1 \text { se o dado do ângulo foi utilizado no } \\
\text { processamento, } 0 \text { caso contrário }\end{array}$ \\
\hline 24 & $\begin{array}{l}\text { Indicador de posição. } 1 \text { se a posição foi utilizada no processamento, } \\
0 \text { caso contrário }\end{array}$ \\
\hline 25 & $\begin{array}{l}\text { Indicador de tempo. } 1 \text { se o tempo foi utilizado no processamento, } \\
0 \text { caso contrário }\end{array}$ \\
\hline
\end{tabular}

Quadro 6: Definição dos Campos do Protocolo UALF 0.

Os parâmetros 15 a 17 deste quadro especificam uma elipse de erro para uma região ao redor de uma determinada localização, dada em latitude/longitude. Este campo representa o ângulo da elipse com orientação horária, onde 0 é o norte; e varia de 0 ㅇ a 180 ‥

De posse destes parâmetros, o software "quebra" o protocolo a fim de separar somente os dados necessários para os cálculos que se seguem. Caso não sejam detectadas descargas atmosféricas ou a distância (d), que corresponde a distância entre o local onde ocorreu a descarga atmosférica e a subestação protegida, seja maior que o raio de atração (RA), o software permanece no modo de prontidão. Por outro lado, caso a distância (d) seja menor que o raio de atração, o software entra no modo de proteção, enviando a ordem para desacoplamento das cargas protegidas da rede elétrica e sua conexão ao no-break - sistema alarmado.

Depois da ocorrência de um alarme o software aguarda quinze minutos para avaliar a existência de risco no local. Se novas descargas não forem registradas dentro do raio de atração durante este período, o software retorna para o modo de prontidão.

Devido à autonomia do no-break utilizado, o sistema não pode permanecer no modo de proteção por um período superior a duas horas. Por esta razão, o software possui um timer que controla este tempo. Caso o sistema permaneça alarmado por cento e 
dezenove minutos, o software envia a ordem para reconexão das cargas protegidas á rede elétrica mesmo que continuem ocorrendo descargas dentro do raio de atração.

Finalmente, vale citar que o software mantém registro de todas as descargas ocorridas dentro do raio de atração, com informações como data, local e intensidade da corrente da descarga.

A figura 2 apresenta a tela do software, acompanhada do relatório de ocorrências. Nesta situação, o offset de captura estava ajustado em $10.000 \mathrm{~m}$, a fim de proporcionar a ocorrências de diversos alarmes.

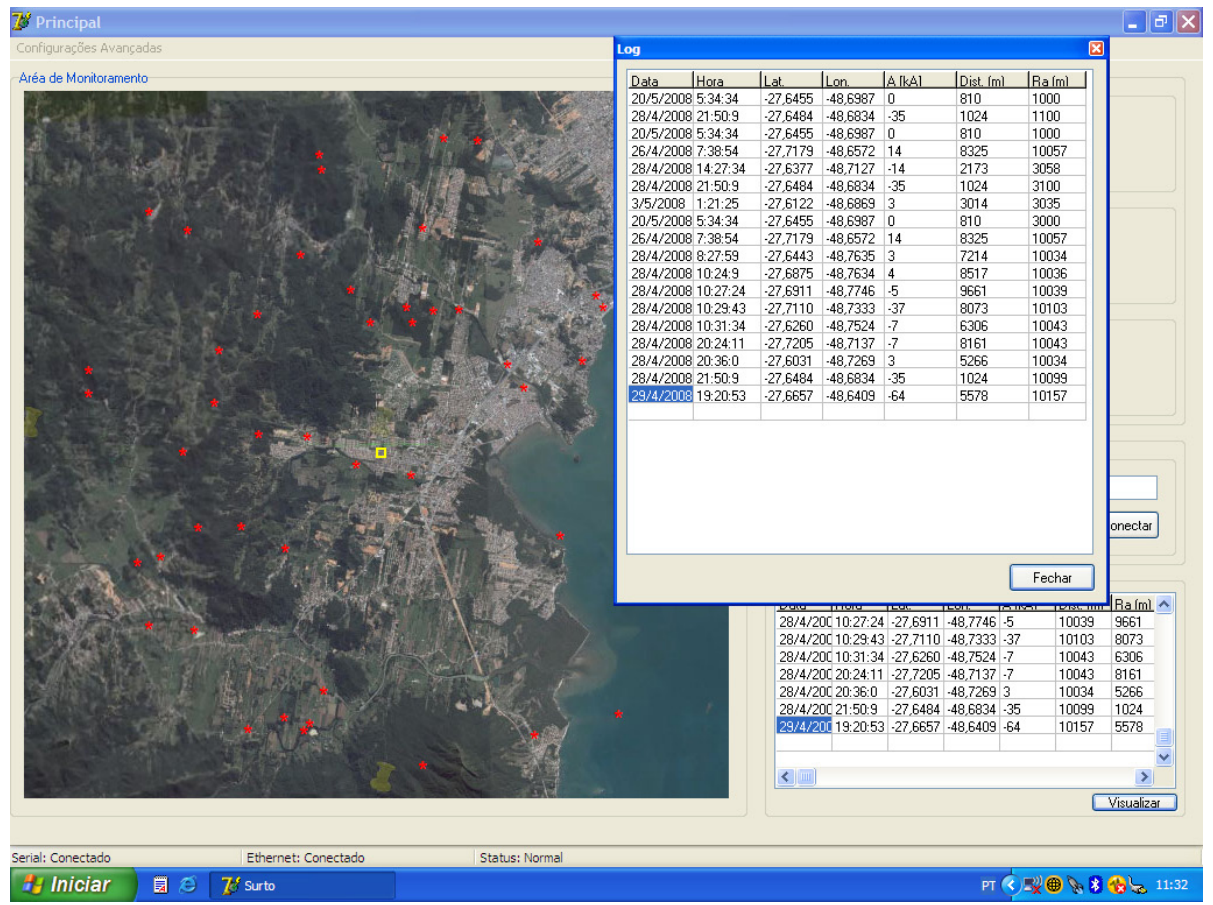

Figura 2: Tela do Software com Relatório de Ocorrências - Offset: 10.000m.

A figura 3 apresenta a tela principal do software com o offset de captura ajustado em $3.000 \mathrm{~m}$. Nesta tela, o quadro amarelo indica a posição da Subestação de Palhoça, os pontos verdes indicam descargas fora do raio de atração e os pontos vermelhos indicam descargas dentro do raio de atração (sistema alarmado). 

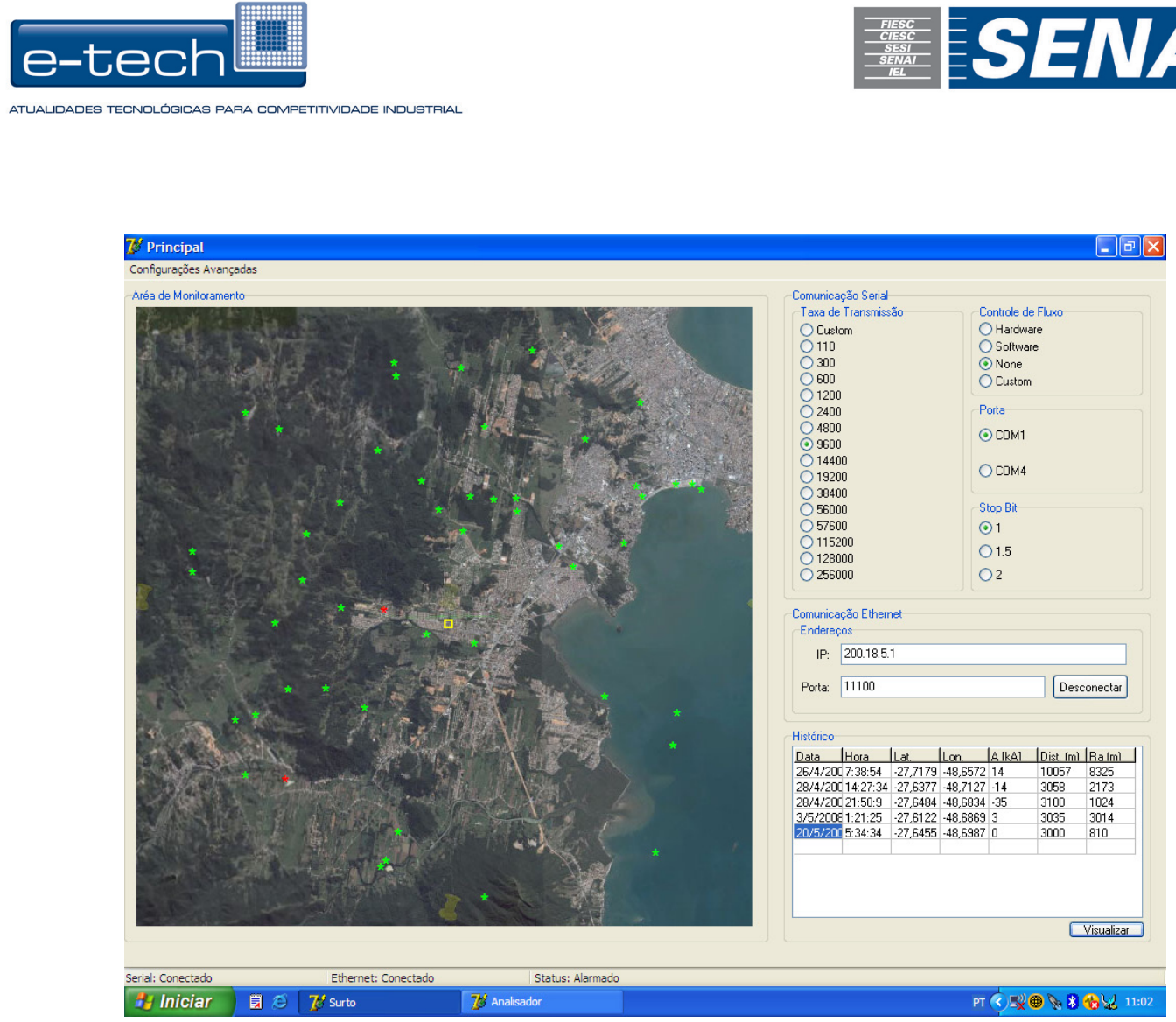

Figura 3: Tela do Software com Relatório de Ocorrências - Offset: 3.000m.

\subsection{HARDWARE DE CONTROLE}

As ordens de comando do software operando nos modos de prontidão ou proteção são interpretadas por um hardware microcontrolado, que faz com que as cargas protegidas sejam conectadas ou desconectadas da rede. Para facilitar o entendimento, do funcionamento do software, este foi dividido em duas partes que serão apresentadas a seguir.

\subsubsection{Interface Microcontrolada}

O sistema eletrônico de interface permite a comunicação entre o software de controle residente em um computador e o sistema de comutação, responsável pelo acionamento do sistema de no-break.

O hardware desta interface está centrado no microcontrolador PIC16F876 e ao receber um sinal da porta serial do software de controle segue as requisições especificadas no projeto, colocando a porta em nível lógico alto ou baixo, além de sinalizar, por meio de led's vermelho e verde o estado do sistema, isto é, se o mesmo encontra-se alarmado ou normal, respectivamente.

Todo o controle é feito por um firmware, cujo fluxograma encontra-se representado na Figura 4. 


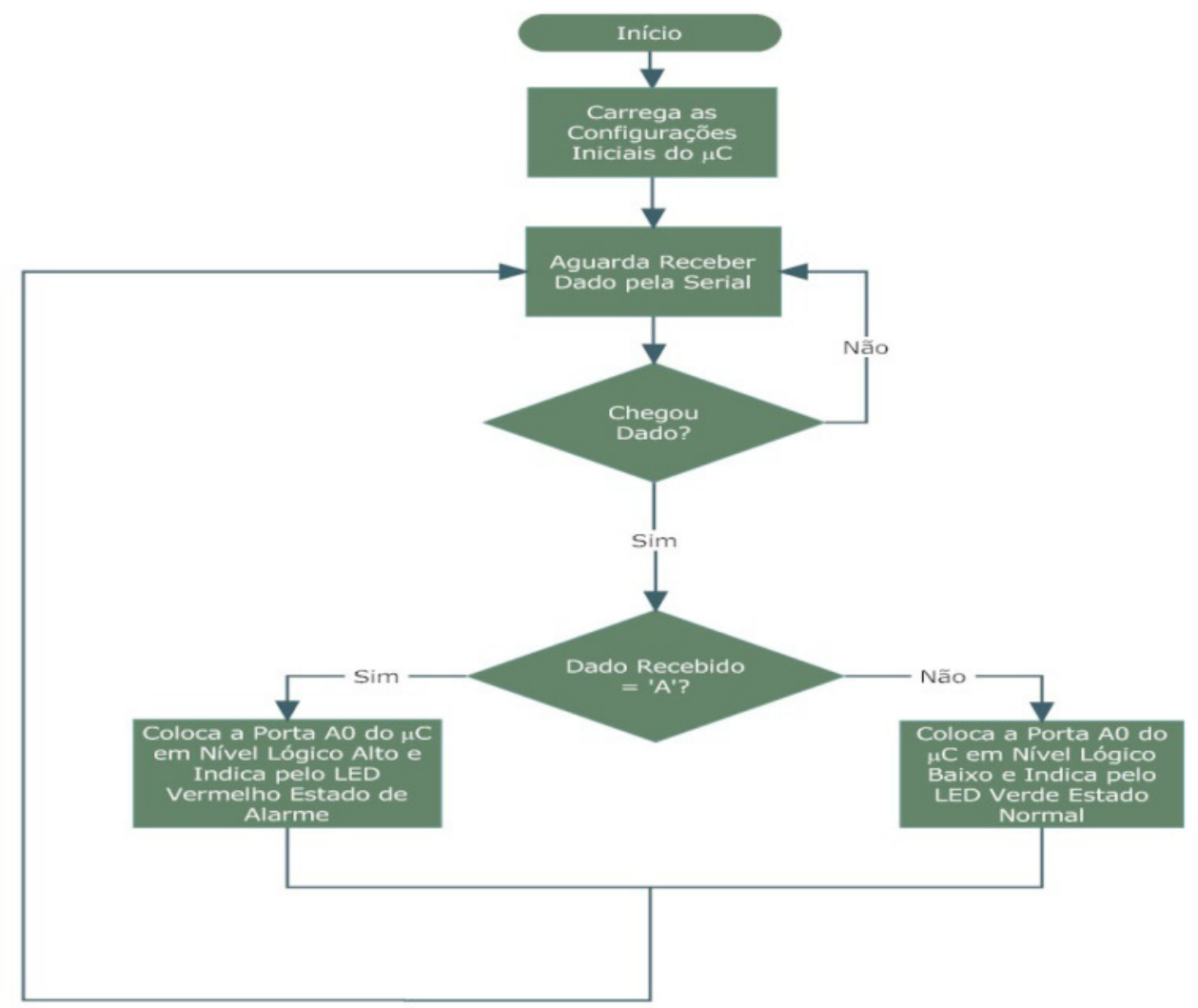

Figura 4: Fluxograma do Firmware.

Como se pode perceber trata-se de uma rotina simples, que mantém o microcontrolador em um loop infinito aguardando as ordens do software de controle. O uso do microcontrolador torna-se interessante porque pode permitir a expansão do sistema em aplicações futuras e a fácil inserção de novas funcionalidades, apenas através da alteração da programação do microcontrolador.

\subsubsection{Sistema de Comutação}

Trata-se de um circuito eletrônico composto basicamente por um comparador não inversor com histerese, que determina se uma tensão é maior ou menor que uma determinada tensão de referência. Assim sendo, sempre que o sinal proveniente da interface microcontrolada superar o valor de referência, o comparador envia um sinal de gate a um transistor tipo Metal Oxide Semiconductor Field Effect Transistor (MOSFET), que foi escolhido pela baixa corrente de comando demandada. O modelo utilizado no protótipo desenvolvido, por questão de disponibilidade foi o IRF740, mas outros modelos equivalentes e para menores correntes podem ser utilizados.

Ao pino de source deste transistor foram conectados cinco relés que permitem comandar o acionamento de até cinco cargas diferentes. No site em estudo foi utilizada somente uma carga, que se caracteriza como um sistema de alimentação ininterrupto do tipo on-line ( no-break on-line), que alimenta os equipamentos a serem protegidos quando $o$ software de controle assim o determinar. 
Finalmente, sempre que a interface enviar um sinal lógico baixo (abaixo da referência estabelecida no projeto), o sistema funciona normalmente a partir da rede elétrica.

\section{VALIDAÇÕES DO SISTEMA PILOTO}

Mediante a obtenção de arquivos de dados reais das descargas atmosféricas via SIDDEM, realizou-se diversos testes de campo para se chegar ao ponto otimizado do protótipo. Das instalações da EPAGRI situada no bairro Itacorubi em Florianópolis os arquivos foram enviados para o computador do projeto na Subestação da Eletrosul em Palhoça.

Diversas alterações e melhorias foram introduzidas no software de controle, destacando o acréscimo ao raio de atração um valor adicional (offset) que permitisse, a critério do usuário, demarcar novas zonas de proteção contra descargas atmosféricas.

Em todas as simulações realizadas o equipamento atuou de maneira satisfatória e deverá ser mantido em funcionamento, por tempo indeterminado, a fim de confirmar sua confiabilidade.

\section{CONCLUSÕES}

Este artigo apresentou as principais etapas e os conceitos mais importantes, utilizados para o desenvolvimento de um dispositivo eletrônico, cuja finalidade é proteger equipamentos eletrônicos de possíveis danos causados por descargas atmosféricas.

Para tanto, utilizou-se a metodologia do raio de atração para o protótipo e tal modelagem foi estruturada numa das teorias de boa aceitação proposta por Rusk (1957), que discorre sobre o cálculo de tensões induzidas por descargas atmosféricas em redes elétricas.

Rusk (1957) apresentou um modelo de canal para descarga atmosférica, onde especificou uma distribuição de cargas e também explicitou uma densidade de corrente no canal. Isto permite o cálculo dos campos eletromagnéticos gerados por este sistema, a partir das equações de Maxwell. Esta teoria foi adotada pelo Institute of Electrical and Electronics Engineers (2004) para estudos de desempenho de linhas de distribuição frente a descargas atmosféricas posteriormente, com aperfeiçoamentos pela Associação Brasileira de Normas Técnicas (2001), na norma NBR5419.

A modelagem matemática que envolve problemas de caminhos aleatórios ou movimentos brownianos, bem como as concepções sobre descargas, encontra-se bem difundidas e absorvidas pelos modernos sistemas de detecção de descargas atmosféricas, nas tecnologias implantadas nos sistemas: Improved Accuracy from Combined Technology (IMPACT) e Système d"Alerte Foudre par Interérométrie Radioélectrique (SAFIR), como apresentado em Naccarato (2006) e Vaisala (2004).

Desta forma, comprova-se que a rede SIDDEM permite, dentre outras, as seguintes aplicações: 
a) mapeamento total das descargas na área de atuação do sistema por intervalos específicos de tempo;

b) monitoramento da concentração de descargas por áreas especificadas pelo usuário e identificação do tipo de descarga (intra-nuvem ou nuvem-terra);

c) mapeamento total da densidade de descarga, medindo a intensidade da tempestade pelo mapeamento da densidade de descargas por $\mathrm{Km}^{2}$ e por minuto. Descargas intra-nuvem ou nuvem-terra podem ser selecionadas via display;

d) definir o contorno da tempestade e trajetória por área de risco para identifica e traçar áreas de risco e as apresenta no display usando código de cores de fácil entendimento;

e) sincronização a evolução da formação da tempestade com alarmes automáticos: - integrando-se o método do raio de atração com os recursos da rede SIDDEM, tem-se os elementos necessários para em tempo real promover um eficaz sistema de alarme e de proteção contra danos provocados em equipamentos eletrônicos, por coordenada geodésica específica;

f) utilização de um valor de offset deu-se pela dificuldade de se conseguir testar com valores reais de descargas atmosféricas, aquelas que caíssem na zona de atração da subestação de Palhoça.

Além disso, adicionou-se ao raio de atração (RA) um valor adicional (offset) com a finalidade de se demarcar novas zonas de proteção, a critério do usuário.

Este procedimento passa a ser um incremento diferencial para novas perspectivas de utilização do projeto pela Eletrosul.

\section{REFERÊNCIAS}

ANDERSON, J.G. Transmission line reference book, $\mathbf{3 4 5}$ kV and Above. California, USA: Electric Power Research Institute, 1982.

ARMSTRONG, H.R., WHITEHEAD, E.R., Field and analytical studies of transmission line shielding. IEEE Transactions on PAS, New York, vol. 87, p. 270 - 281, Jan. 1968.

ASSOCIAÇÃO BRASILEIRA DE NORMAS TÉCNICAS. NBR-5419: proteção de estruturas contra descargas atmosféricas. Rio de Janeiro: 2001. 
BROWN, G.W., WHITEHEAD, E.R., Field and analytical studies of transmission line shielding - II. IEEE Transactions on PAS, New York, vol. 8, p. 617-626, 1969.

ERIKSSON, A. J. An Improved Electrogeometric Model for Transmission Line Shielding Analysis. IEEE Trans. on Power Delivery, New York, vol.PWRD-2,no.3, p. 871-886, jul.1987.

INSTITUTE OF ELECTRICAL AND ELECTRONICS ENGINEERS. Guide for improving the lightning performance of electric power overhead distribution lines. New York: IEEE STD, 2004.

LOVE, E.R. Improvements on lighting stroke modeling and applications to design of EHV and UHV transmission ILines, MSC. Thesis, Colorado University, USA, 1973.

NACCARATO, K. P. Análise das características dos relâmpagos na região sudeste do Brasil. 2005. 362 f. Tese (Doutorado em Geofísica Espacial) - Instituto de Pesquisas Espaciais, São José dos Campos, 2006.

RUSK, S. Induced lightning over-voltages on power transmission lines with special reference to the over protection of low voltage networks. Tese (Doutorado) Göteborg, Sweden, 1957.

VAISALA. IMPACT ESP (Model 141 TESP): user's guide. Versão 1.3(M210416EN-A). Tucson, 2004.

VISACRO FILHO, Silvério. Descargas atmosféricas: uma abordagem de engenharia. São Paulo: Artilibe, 2005.

Originais recebidos em: 30/09/2009

Texto aprovado em: 12/04/2010 


\section{SOBRE OS AUTORES}

Doutor em Engenharia Elétrica pela Universidade Federal de Santa Catarina
(2004), tendo também obtido o título de Mestre em Engenharia elétrica na
mesma instituição no ano de 1997. Sua graduação, em Engenharia Elétrica,
foi concluída em 1995 na Fundação Universidade Regional de Blumenau.
Atualmente é coordenador do curso de Engenharia Elétrica - Telemática da
Universidade do Sul de Santa Catarina, atuando também como professor no
curso superior de tecnologia do SENAl/SC em São José. Tem experiência na
área de Engenharia Elétrica, com ênfase em Eletrônica Industrial, atuando
principalmente nos seguintes temas: eletrônica de potência, lâmpada de vapor
de sódio de alta pressão, alto fator de potência, lâmpada fluorescente e reator.
E-mail: Anderson.André@unisul.br
André

\begin{tabular}{|l} 
Possui graduação em Engenharia Elétrica pela UERJ, especialização em \\
Confiabilidade de Sistemas de Potência pela UNIFEI, mestrado pela UFSC e \\
doutorado pela Engenharia de Produção da UFSC. \\
Atualmente é docente da UNISUL- Universidade do Sul de Santa Catarina, nos \\
cursos de engenharia elétrica, ciência da computação e sistema de informações. \\
Trabalhou 22 anos como engenheiro de operação/manutenção da Eletrosul \\
Centrais Elétricas S.A. Tem experiência em Transmissão e Geração de energia, \\
atuando nos temas: engenharia do conhecimento, operação, confiabilidade e e \\
manutenção de sistemas de energia. Dedica-se, também à pesquisa na área de \\
energia e coordena o GPEAR - Grupo de Pesquisas em Energias Alternativas e \\
Renováveis da Unisul. \\
E-mail: Joao.Alkaim@unisul.br
\end{tabular}

\begin{tabular}{|l} 
Formação: graduação (1987) e mestrado (1994) em Engenharia Elétrica e \\
doutorado em Engenharia de Produção (2004) pela Universidade Federal de \\
Santa Catarina. \\
Vínculo profissional: Professor efetivo da Universidade do Estado de Santa \\
Catarina - Udesc e da Universidade do Sul de Santa Catarina - Unisul, na qual \\
exerce o vice-coordenador do curso de Engenharia Elétrica Telemática. É \\
Conselheiro do CREA-SC, onde Coordena a Câmara Especializada em Engenharia \\
Elétrica e a Câmara Nacional da área, pelo CONFEA. \\
Julibio David \\
Ardigo \\
Administração e Engenharia, principalmente em infra-estrutura de tecnologia \\
e e-Business \\
E-mail: Julibio.Ardigo@unisul.br
\end{tabular}




\begin{tabular}{|l|l|} 
Eletrotécnico e engenheiro eletricista, possui mestrado pela UFSC (2002) \\
e doutorado com duplo-diploma pela Ecole Centrale de Lyon e UFSC (2006). \\
Realizou seu primeiro estágio de pós-doutoramento industrial no ano de \\
2006 na Schneider Electric (INPG/IDEA/GIE), onde ajudou a desenvolver uma \\
nova tecnologia para esta empresa. Em 2008 concluiu o seu segundo estágio \\
de pós-doutoramento no LMAG/USP. Atua como professor na UNISUL. Tem \\
experiência em engenharia; otimização e análise de sensibilidade na concepção \\
de dispositivos e sistemas; estudos de viabilidade de negócios e gerenciamento \\
de projetos.
\end{tabular}

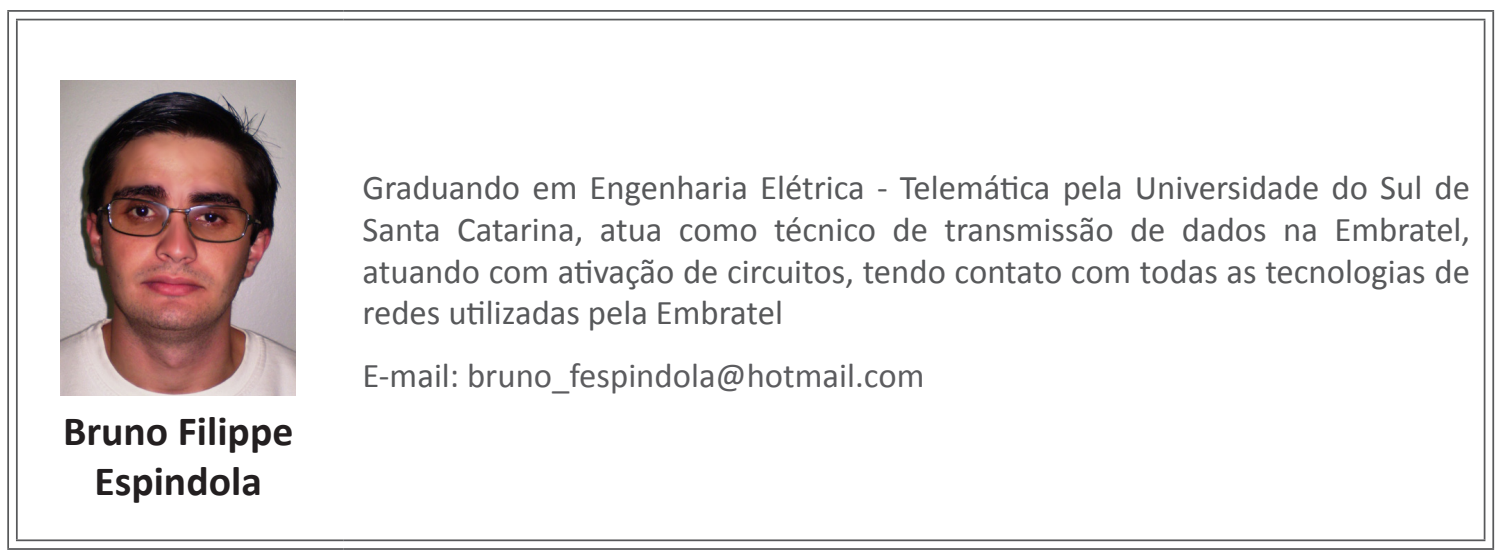

